\title{
Distribution network reconfiguration using feasibility-preserving evolutionary optimization
}

\author{
Alberto LANDEROS ${ }^{1}$, Slawomir KOZIEL ${ }^{1,2}$, Mohamed F. ABDEL-FATTAH ${ }^{1}$
}

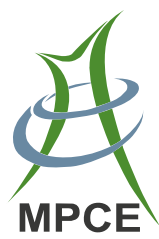

\begin{abstract}
Distribution network reconfiguration (DNR) can significantly reduce power losses, improve the voltage profile, and increase the power quality. DNR studies require implementation of power flow analysis and complex optimization procedures capable of handling large combinatorial problems. The size of distribution network influences the type of the optimization method to be applied. Straightforward approaches can be computationally expensive or even prohibitive whereas heuristic or meta-heuristic approaches can yield acceptable results with less computation cost. In this paper, a customized evolutionary algorithm has been introduced and applied to power distribution network reconfiguration. The recombination operators of the algorithm are designed to preserve feasibility of solutions (radial structure of the network) thus considerably reducing the size of the search space. Consequently, improved repeatability of results as well as lower overall computational complexity of the optimization process have been achieved. The optimization process considers power losses and the system voltage profile, both
\end{abstract}

CrossCheck date: 6 September 2018

Received: 12 February 2018/ Accepted: 6 September 2018/Published online: 13 December 2018

(C) The Author(s) 2018

$\triangle$ Mohamed F. ABDEL-FATTAH

abdelfattah@ru.is

Alberto LANDEROS

alberto16@ru.is

Slawomir KOZIEL koziel@ru.is

1 School of Science and Engineering, Reykjavik University, 101 Reykjavik, Iceland

2 Faculty of Electronics, Telecommunications and Informatics, Gdansk University of Technology, 80-233 Gdańsk, Poland aggregated into a scalar cost function. Power flow analysis is performed with the Open Distribution System Simulator, a simple and efficient simulation tool for electric distribution systems. Our approach is demonstrated using several networks of various sizes. Comprehensive benchmarking indicates superiority of the proposed technique over state-of-the-art methods from the literature.

Keywords Distribution network reconfiguration, Feasibility-preserving evolutionary optimization, Power loss reduction, Radial networks, Voltage profile

\section{Introduction}

Power losses in distribution systems may be significant and may negatively affect the economics of electric power distribution networks [1]. Consequently, it is of interest to study the reduction of losses through techniques such as distribution network reconfiguration (DNR). The topology of power distribution systems is typically radial, whereas transmission systems can operate in loop or radial configurations [2].

Radial distribution systems often feature sectionalizing switches and tie switches, mainly used for fault isolation, power supply recovery and system reconfiguration.

These switches allow for reconfiguring the topology of the network, with the objectives being reduction of power losses, load balancing, and improvement of voltage profile and system reliability [3]. A large number of permutations, resulting from all possible switch configurations, make the network reconfiguration task a complex and non-linear combinatorial problem, particularly for large systems. Due to the power flow calculations involved [4], the incurred computational cost can be considerable. 
The first example of DNR for power loss reduction [5] was a branch and bound search with all tie lines initially closed, thus creating a meshed system; subsequently switch opening was done until a radial configuration was achieved. Similarly, a method was proposed in [6], where the network was initially meshed and the switches were ranked based on the current carried. The top-ranked switch was opened and the power flow calculation was carried out. The process was repeated until the system was radial. A branch exchange was performed wherever a loop had been identified. The configuration with lower power losses was kept.

In [7], a generalized approach was proposed in which the tie line with the highest voltage difference was closed and the neighbouring branch was opened in the loop formed, leading to a reduction of power losses. Reference [8] implemented DNR through node-depth encoding (NDE), which improved the performance of evolutionary algorithms and power flow algorithms. On average, $27.64 \%$ in loss reduction was obtained on 30880 buses in a 5166-switch system.

The DNR problem is, in general, a multi-modal one, and hence computational intelligence algorithms are normally more appropriate, even though they generally do not converge to the global optima for larger size systems.

In [2], DNR was performed using the cuckoo search algorithm (CSA) on 33-, 69- and 119-node distribution systems and compared to methods presented in other works. The power loss values presented for the 33- and 69-node systems were the same as those implementing continuous genetic algorithm (CGA) and particle swarm optimization (PSO); the losses were smaller than those applying fireworks algorithm (FWA) [9], genetic algorithm (GA), refined GA (RGA), improved tabu search (ITS), harmony search algorithm (HSA) [10], ant colony (AC) algorithm, and improved adaptive imperialist competitive algorithm (IAICA) [11]. For the 119-node system, CSA and CGA presented the same loss level, where FWA and HSA presented the lowest.

For larger distribution systems (with hundreds or thousands of buses), DNR can be computationally expensive and requires unacceptable computing time.

Power flow analysis is an essential task of DNR, necessary to evaluate the status of the distribution network before and after reconfiguration. Thus, it serves as a reference to determine the effects of network reconfiguration on quantities of interest (e.g., line power losses and/or voltage profile). Due to the multi-combinatorial nature of DNR, a large number of power flow analyses are required [12].

The usual radial topology of distribution networks has driven power flow analysis to be done with techniques such as the forward-backward sweep ladder method [13], because traditional methods such as the decoupled version of Newton Raphson have shown convergence problems leading to undesirable solutions [14].

The Open Distribution System Simulation (OpenDSS) is an efficient and fast simulation tool for distribution network analysis. It can perform power flow analysis although it evolved as a harmonic flow analysis tool [15]. It handles not only radial networks but arbitrarily-meshed, multiphase networks. The power flow solving methods implemented by OpenDSS are based on the "current injection mode". The use of the Newton method enables OpenDSS to be a fast and robust power flow analysis tool [16].

In this paper, a customized evolutionary algorithm has been proposed for solving a DNR problem for radial networks. The major differences between conventional evolutionary algorithms and the proposed one are in dedicated recombination operators that embed specific knowledge of the problem. By enforcing feasibility of solutions, particularly maintaining radial network structure at all stages of the process, a considerable reduction of the search space size is obtained. This leads to faster convergence of the optimization process, as well as improved repeatability of the results, based on the numerical studies carried out for the 33-, 69-, and 119-bus test problems. More importantly, comprehensive benchmarking indicates superiority of the proposed technique over state-of-the-art algorithms reported in the literature.

\section{Problem formulation}

\subsection{Objective function}

Network reconfiguration is realized by changing the state of the switches (open/close). The objectives of this change in the system are to minimize the power losses and to improve the voltage profile/index. In more rigorous terms, the objective is to minimize the expression in (1) [9]:

$F=\Delta P_{\text {loss }}^{\text {ratio }}+\Delta V_{\mathrm{D}}$

where $\Delta P_{\text {loss }}^{\text {ratio }}$ is the ratio of the total power loss in the branches after reconfiguration $\Delta P_{\text {loss }}^{\text {rec }}$ to the initial power losses before reconfiguration $\Delta P_{\text {loss }}^{\text {init }}$, as in (2); $\Delta V_{\mathrm{D}}$ is the voltage variation index and calculated by finding the maximum voltage drop for all buses using the ratios of bus voltages $V_{i}$ to the reference source voltage $V_{1}$, as in (3):

$$
\begin{aligned}
& \Delta P_{\text {loss }}^{\text {ratio }}=P_{\text {loss }}^{\text {rec }} / P_{\text {loss }}^{\text {init }} \\
& \Delta V_{\mathrm{D}}=\max _{i=1,2, \cdots, N_{\text {bus }}}\left|1-V_{i} / V_{1}\right|
\end{aligned}
$$

In (1), one takes into account the change in losses after the reconfiguration as well as the deviation of voltage in 
relation to the base voltage, and aims to minimize both parameters. The constraint is to maintain the radial architecture of the network, for supplying all load points.

The network power losses are calculated by adding up the losses in all active network branches, as in (4), where $P_{i}$ and $Q_{i}$ represent the active and reactive power flow out of bus $i$, respectively; $R_{i}$ is the resistance of line segment $i ; V_{i}$ is the voltage at the $i^{\text {th }}$ bus; $N_{\mathrm{br}}$ is the number of active branches, given by (5):

$$
\begin{aligned}
& P_{\text {loss }}=\sum_{i=1}^{N_{\text {br }}} R_{i} \frac{P_{i}^{2}+Q_{i}^{2}}{V_{i}^{2}} \\
& N_{\text {br }}=N_{\text {bus }}-1
\end{aligned}
$$

\subsection{Power flow}

OpenDSS is utilized to carry out power flow simulations of the test distribution systems described in Section 4. For the radial circuits, as the considered ones here, OpenDSS exhibits convergence characteristics similar to those of forward-backward sweep methods [15].

The test distribution systems are modeled as power delivery elements (power lines) and power conversion elements (loads) in the OpenDSS environment. The OpenDSS script-driven simulation engine has a component object model (COM) which allows MATLAB command to access OpenDSS features as illustrated in Fig. 1. Some of the commands include switch operations, power flow execution, results extraction, etc.

Switching operations require a switch control assigned to every branch in the network; given sectionalizing switches $N_{\mathrm{sec}}$ and tie switches $N_{\mathrm{ts}}$, the total number of controlled switches $N_{\mathrm{s}}$ is given by (6):

$N_{\mathrm{s}}=N_{\mathrm{sec}}+N_{\mathrm{ts}}$

The switches status is expressed by a binary string $x$ with zeros representing open switches and ones representing closed switches.

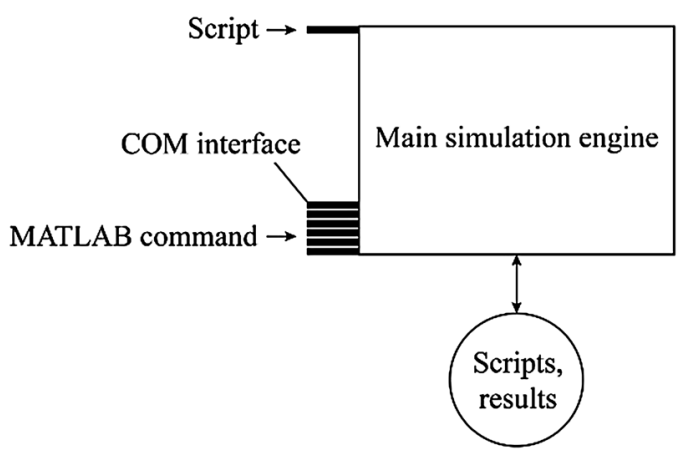

Fig. 1 Main simulation engine of OpenDSS accessible to MATLAB command

\section{Optimization methodology}

In this section, the proposed optimization algorithm is outlined. It is referred to as feasibility-preserving evolutionary optimization (FPEO). Similar to the previous works in the literature, as in [2], a population-based metaheuristic method is selected due to its ability to perform global search. This is necessary because DNR is intrinsically a multi-modal problem. The algorithm is tailored to the task at hand, in particular, a radial network configuration is maintained throughout the optimization run. The operation and performance of the method is demonstrated in Section 4.

\subsection{Representation}

The solutions are represented as binary strings with zeros corresponding to open switches (no connection) and ones corresponding to close switches (existing connections).

Given $N_{\mathrm{br}}$ and $N_{\mathrm{ts}}$, the number of possible network configurations is $C\left(N_{\mathrm{br}}, N_{\mathrm{ts}}\right)$ which is large and grows quickly with both $N_{\mathrm{br}}$ and $N_{\mathrm{ts}}$. At the same time, the number of radial configurations, equal to the number of the spanning trees $\tau(G)$ of the network graph $G$, is much smaller. For example, for the 119-bus system in [17], the numbers are $2.4 \times 10^{19}$ and $4 \times 10^{15}$ (the latter estimated using the matrix-tree theorem [18]). Consequently, it is beneficial to maintain feasibility of solutions throughout the entire optimization run. In the proposed algorithm, it is realized by appropriate definition of the recombination and mutation operators.

\subsection{Algorithm flow}

The proposed algorithm follows the basic steps of the generational evolutionary algorithms. The flow diagram of the proposed algorithm is shown in Fig. 2, where $P$ stands for the population.

The algorithm uses binary tournament selection [19]. It also features elitism and adaptive adjustment of mutation probability based on population diversity. The elitism works by replacing the first individual of the newly created population by the best individual found so far, which allows it to bypass the selection and recombination procedures, and, thereby, to preserve it across the algorithm iterations. Diversity is measured as the average standard deviation of solution components. The vector $F$ of cost function values for the population is used by the selection procedure. The two critical (and novel) components of the algorithm are mutation and crossover operators, both 


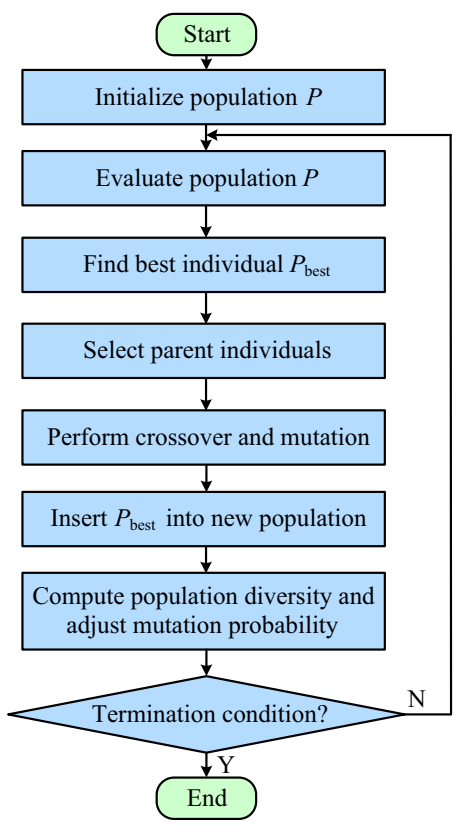

Fig. 2 Flow diagram of proposed algorithm

designed to maintain radial structure of the network. They are briefly described in the following two subsections.

\subsection{Feasibility-preserving mutation operator}

The mutation operator is supposed to introduce a small random change in the network configuration. Here, it is implemented as follows. First, one of the open switches is randomly selected and closed creating a loop in the network. Then, the loop created this way is identified. Finally, one of the connections on the loop is randomly selected to be opened. As a result, the network is transformed from one radial configuration to another. The mutation operator has also been explained in Fig. 3.

\subsection{Feasibility-preserving crossover operator}

The crossover operator, similarly as the mutation one, is designed to maintain radial configuration of the network. The first step is a conventional uniform crossover [20], where components of the offspring $\boldsymbol{x}$ are randomly selected from one of the two parent individuals. In the second step, a repair procedure is launched according to the flow diagram shown in Fig. 4, where $G(\boldsymbol{x})$ represents the network graph corresponding to the configuration $\boldsymbol{x}$.

In the above algorithm, connectivity of the network graph is first checked. In case the graph is not connected, subsequent switches are closed until connectivity is achieved. At this step, only those switches that do not lead to creating loops can be closed. Otherwise, subsequent connections are opened until the network graph becomes a

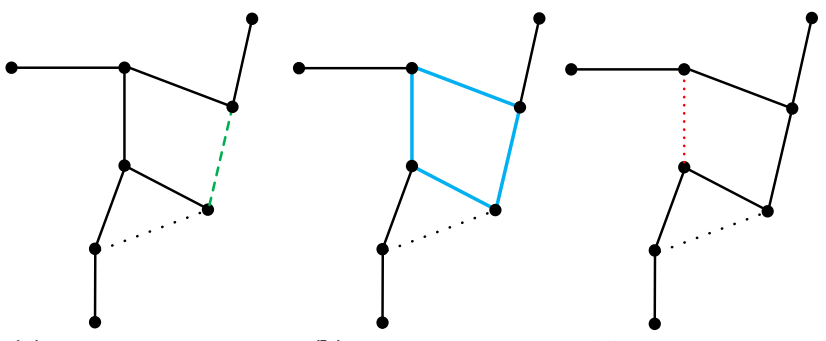

(a) Part of radial network with a switch randomly closed (green dashed line) (b) A loop created by closing the switch (blue solid line) (c) A switch along the loop randomly opened to retain radial configuration

(red dotted line)
Fig. 3 Operation of feasibility-preserving mutation operator

tree which corresponds to a radial network configuration. While modifying the individual, the switches that have already been tried out are stored in order to avoid unnecessary repetitions. The flow diagram of the crossover operator is shown in Fig. 5.

It should be emphasized that due to the repair procedure, the network modification made by a crossover operation can be quite extensive, especially in the initial stages of the optimization run when diversity of the population is large. Consequently, a low crossover probability is used (here, 0.2 ), which is more advantageous as indicated by the initial experiments.

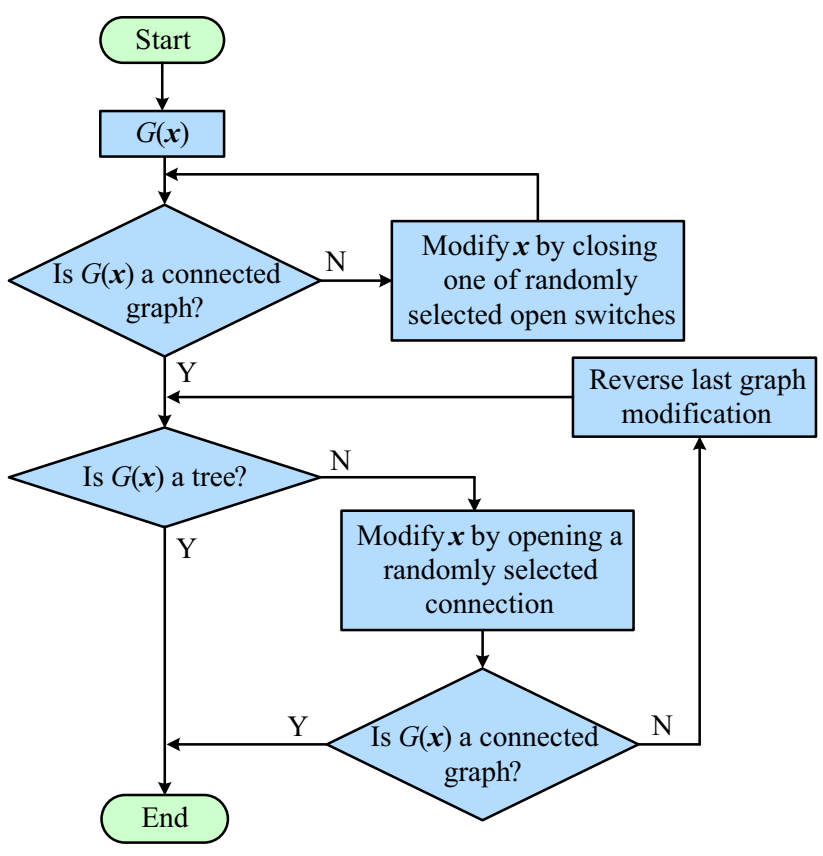

Fig. 4 Flow diagram of repair procedure 


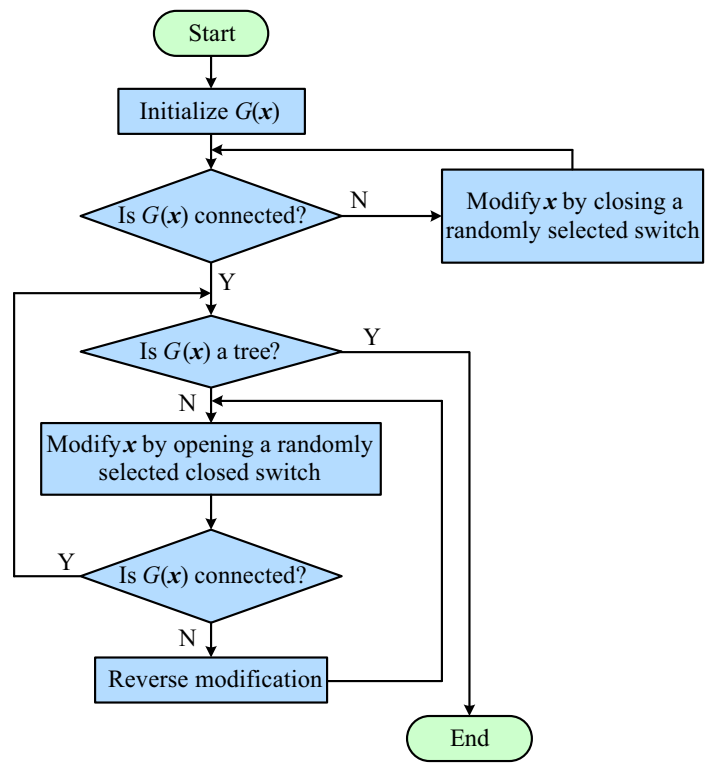

Fig. 5 Flow diagram of feasibility-preserving crossover operator

\section{Numerical results and benchmarking}

In this section, the FPEO algorithm introduced in Section 3 has been comprehensively validated using 3 standard test networks $[16,17,21]$, consisting of 33, 69 and 119 buses, presented in Figs. 6, 7 and 8, respectively. The network topologies are briefly discussed in Section 4.1. Numerical results are presented in Section 4.2 along with comparison to state-of-the-art methods from the literature.

\subsection{Test cases}

The systems are assumed to be balanced and hence single phase representation is sufficient.

The initial conditions of the systems, obtained from the power flow method outlined in Section 2.2, are described in Table 1, where $V_{\min }$ is the minimum voltage found in the system, for all busses, and the "Initial loss" is the total system power losses. For power flow purposes, the L-N base voltage level for the 33- and 69-bus systems is set to be $12.66 \mathrm{kV}$, while for the 119-bus system, the base volt-

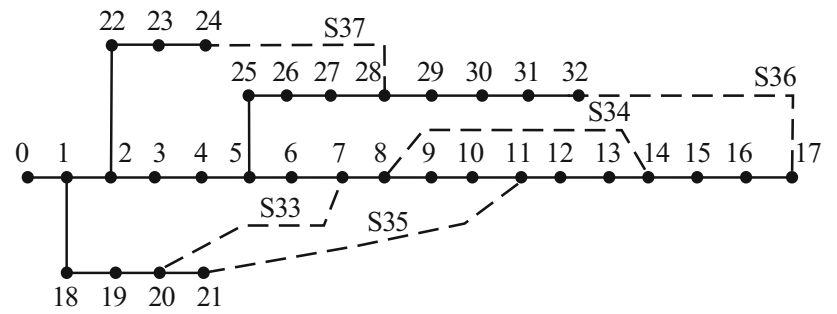

Fig. 6 33-bus distribution system

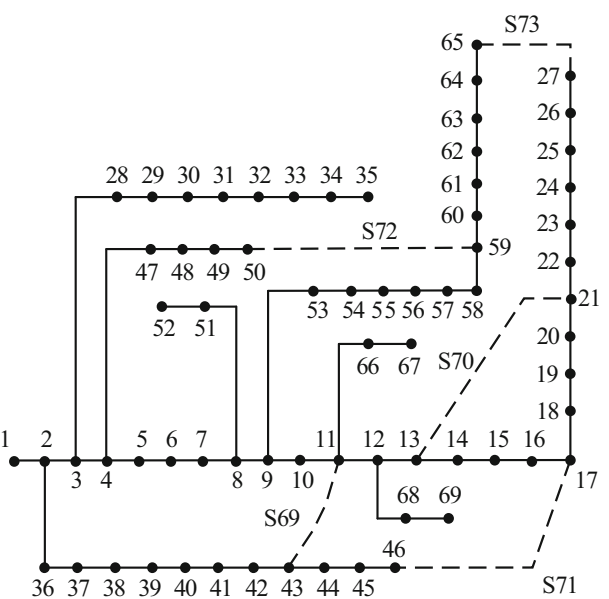

Fig. 7 69-bus distribution system

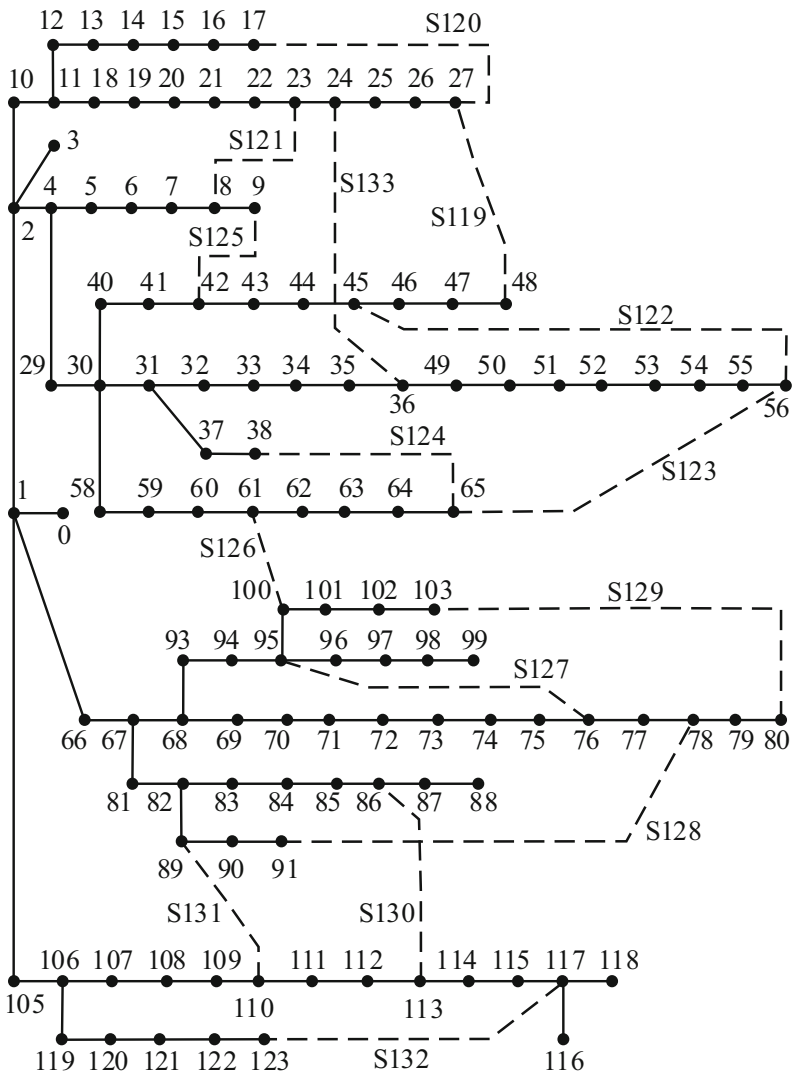

Fig. 8 119-bus distribution system

age level set is $11 \mathrm{kV}$; a tolerance threshold of 0.005 is set.

The initial topologies of the considered systems are shown in Figs. 6, 7 and 8. Each bus is indexed for easy identification. The discontinuous lines represent the tie lines, and each is labeled with the switch number of the switch that operates the tie line. 
Table 1 Initial parameters of test distribution networks

\begin{tabular}{lcll}
\hline Test case & Initial loss $(\mathrm{kW})$ & $V_{\min }$ (p.u.) & Initial open switches \\
\hline 33-Bus system [16] & 200.745 & 0.9107 & $33,34,35,36,37$ \\
69-Bus system [21] & 223.725 & 0.9094 & $69,70,71,72,73$ \\
119-Bus system [17] & 1298.100 & 0.8667 & $119,120,121,122,123,124,125,126,127,128,129,130,131,132,133$ \\
\hline
\end{tabular}

Based on the fact that the voltage source operates as a Thevenin equivalent source, the complex impedance, set during the modelling in the OpenDSS environment, is the impedance of the first line connected to the voltage source bus.

\subsection{Results and benchmarking}

The proposed algorithm has been executed using the following setup: population size 10 , crossover probability 0.2 , and mutation probability 0.2 . It should be noted that these values are different from typical ones utilized in evolutionary algorithms (e.g., 0.5 to 0.9 for crossover, and 0.01 to 0.05 for mutation). Extensive initial experiments conducted for various values of the control parameters (e.g., crossover rate between 0.1 and 0.5 , mutation rate between 0.05 and 0.2 ) indicate that the values utilized above provide the best results, however, the algorithm works relatively well also for other setups within the aforementioned ranges. In particular, the cost function averaged over 20 independent runs may increase up to a few percent as compared to the algorithm using the control parameter values.

As already explained in Section 3.4, the dedicated crossover operator designed for FPEO is rather disruptive so that lower crossover probability has to be used. Suitable values of this and other parameters such as population size and mutation probability have been obtained through initial experiments. In particular, higher mutation probability is necessary in order to maintain sufficient population diversity (although a particular value of 0.2 is not critical because it is adaptively adjusted during the optimization run).

Maximum number of function evaluations was set to 500, 1000, and 5000 for 33-, 69- and 119-bus systems, respectively, which corresponds to 50, 100, and 500 algorithm iterations. The results are shown in Tables 2, 3 and 4, for 33-, 69- and 119-bus systems, respectively. In Tables 2, 3 and 4, different power losses for the same network configuration in some instances are due to different simulation software packages used by various authors; $N_{\text {evals }}$ is the number of cost function evaluations, equal to the

Table 2 Statistics of the optimization results of the 33-bus test network

\begin{tabular}{|c|c|c|c|c|c|c|c|}
\hline Method & $N_{\text {evals }}$ & Best $(\mathrm{kW})$ & Average $(\mathrm{kW})$ & Worst (kW) & Standard deviation & Open switches & Fitness \\
\hline FPEO & 500 & 140.3350 & 140.3350 & 140.3350 & 0.00 & $7,9,14,28,32$ & 0.7607 \\
\hline CSA [2] & 3000 & 139.8476 & N/A & N/A & N/A & $7,9,14,32,37$ & 0.7618 \\
\hline FWA [9] & 1000 & 140.3350 & 147.0200 & 157.2430 & 5.39 & $7,9,14,28,32$ & 0.7607 \\
\hline HSA [10] & 2500 & 142.8780 & 153.8200 & 197.0100 & 11.28 & $7,10,14,36,37$ & 0.7810 \\
\hline RGA [10] & N/A & 139.8476 & 166.5100 & 200.3400 & 13.34 & $7,9,14,32,37$ & 0.7618 \\
\hline ITS [10] & 600 & 142.8780 & 165.1000 & 198.2200 & 12.11 & $7,9,14,36,37$ & 0.7810 \\
\hline GA [10] & 21000 & 139.8476 & 167.8200 & 204.6800 & 14.54 & $7,9,14,32,37$ & 0.7618 \\
\hline
\end{tabular}

Table 3 Statistics of the optimization results of the 69-bus test network

\begin{tabular}{lcllllrl}
\hline Method & $N_{\text {evals }}$ & Best $(\mathrm{kW})$ & Average $(\mathrm{kW})$ & Worst $(\mathrm{kW})$ & Standard deviation & Open switches & Fitness \\
\hline FPEO & 1000 & 98.9299 & 98.9299 & 98.9299 & 0.00 & $14,55,61,69,70$ & 0.4492 \\
CSA [2] & 3000 & 98.9418 & N/A & N/A & N/A & $14,57,61,69,70$ \\
GA [11] & 900 & 98.9418 & 101.3400 & 104.7300 & N/A & 0.4993 \\
AC [11] & 900 & 99.1225 & 103.1800 & 110.2800 & N/A & $14,57,61,69,70$ \\
IAICA [11] & 900 & 98.9418 & 100.5700 & 104.2500 & N/A & $12,55,61,69,70$ & 0.4993 \\
\end{tabular}


Table 4 Statistics of the optimization results of the 119-bus test network

\begin{tabular}{llllllll}
\hline Method & $N_{\text {evals }}$ & $\begin{array}{l}\text { Best } \\
(\mathrm{kW})\end{array}$ & $\begin{array}{l}\text { Average } \\
(\mathrm{kW})\end{array}$ & $\begin{array}{l}\text { Worst } \\
(\mathrm{kW})\end{array}$ & $\begin{array}{l}\text { Standard } \\
\text { deviation }\end{array}$ & Open switches & Fitness \\
\hline FPEO & 5000 & 856.8000 & 861.1900 & 865.5850 & 1.90 & $24,26,35,40,43,51,59,72,75,96,98,110,122,130,131$ & 0.7300 \\
CSA [2] & 15000 & 856.8000 & N/A & N/A & N/A & $24,26,35,40,43,51,59,72,75,96,98,110,122,130,131$ & 0.7300 \\
FWA [9] & 3000 & 856.8000 & 887.5300 & 942.6300 & 29.58 & $24,26,35,40,43,51,59,72,75,96,98,110,122,130,131$ & 0.7300 \\
CGA [2] & 15000 & 856.8000 & N/A & N/A & N/A & $24,26,35,40,43,51,59,72,75,96,98,110,122,130,131$ & 0.7300 \\
PSO [2] & 1000 & 898.6068 & N/A & N/A & N/A & $9,23,35,43,52,60,71,74,82,96,99,110,120,122,131$ & 0.7626 \\
MTS [22] & N/A & 884.9000 & N/A & N/A & N/A & $23,27,33,40,43,49,52,62,72,74,77,83,110,126,131$ & 0.7493 \\
\hline
\end{tabular}

population size times number iterations; N/A means that relevant data has not been provided.

FPEO has been executed 20 times in order to obtain meaningful statistical data. The results obtained for the 33-bus test network are compared to those presented in $[2,9,10]$; configurations presented by the specified references are simulated using the power flow method described in Section 2.2.

As shown in Table 2, the power losses are slightly higher than those presented in [2], RGA in [15] and GA in [10], however, the fitness function value of the proposed method exhibits the lowest value. The reason is objective aggregation, as indicated in (1), i.e. the objective function value is a composition of the power losses and the bus voltage index.

It should be emphasized that repeatability of the results is excellent for the proposed FPEO: e.g. in this case the algorithm returns the globally optimum results in all runs (i.e., zero standard deviation). This is not the case for the benchmark methods as shown in Table 2. Thus, the reliability of FPEO significantly exceeds reliability of other techniques.

For the 69-bus test network, the results are consistent with those obtained for the 33-bus network, i.e., globally optimum results have been obtained in all algorithm runs as shown in Table 3. For the 119-bus test network, the results are presented in Table 4 . The configuration obtained is the same as that of CSA in [2], FWA in [9] and CGA in [2]. The results for PSO [2] and modified tabu search (MTS) [22] are also presented. As indicated in Table 4, the statistical data reflects a clear indication that FPEO obtains global optima for vast majority of runs (19 out 29 ; the standard deviation is very low).

Figures 9, 10 and 11 show the optimization history for the 3 considered test cases. In Figs. 9, 10 and 11, red lines indicate objective function value versus iteration index for 20 algorithm runs; the black line is an average value. The optimum value is obtained for all but one algorithm runs with the algorithm being virtually converged after 35-40 iterations (which corresponds to 350-400 system

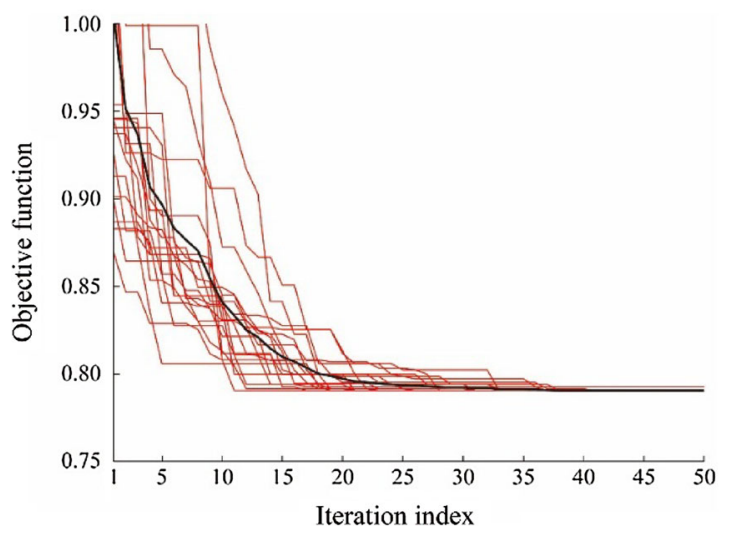

Fig. 9 Optimization history for 33-bus system

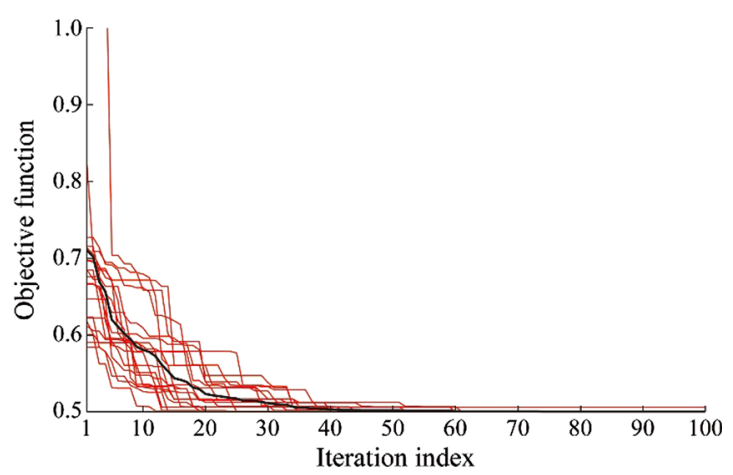

Fig. 10 Optimization history for 69-bus system

simulations) for Fig. 9 (cf. Table 2), and after 50-60 iterations (which corresponds to 500-600 system simulations) for Fig. 10 (cf. Table 3). The optimum value has been obtained for most of the algorithm runs for Fig. 11 (cf. Table 4).

It can be observed that the evolution of the objective function (versus iteration index) is consistent for all algorithm runs thus confirming its robustness. The voltage profiles and power losses before (initial) and after the optimization (optimized) are shown in Figs. 12 and 13, respectively, which confirm the voltage profile improvement. After the optimized reconfiguration, the minimum 


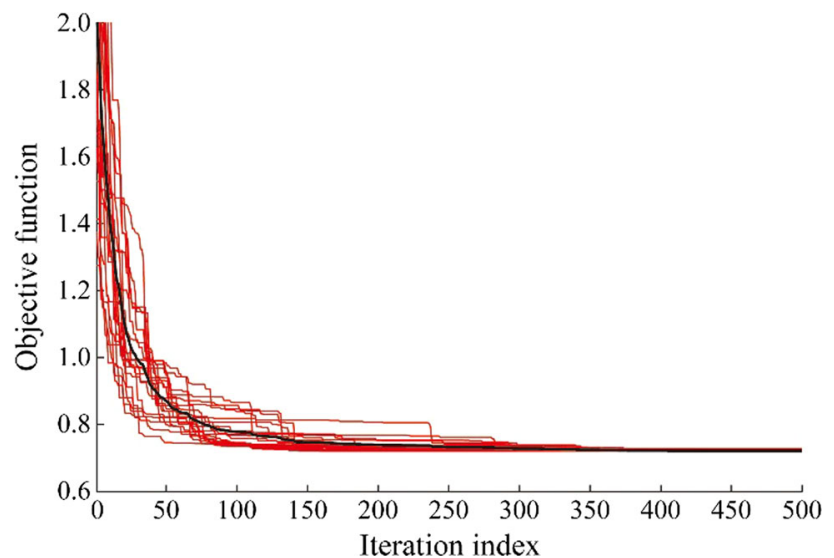

Fig. 11 Optimization history for 119-bus system

p.u. voltage is increased to $0.9383,0.9430$ and 0.9305 p.u. for 33-, 69- and 119-bus networks respectively.

\subsection{Discussion}

FPEO has been demonstrated to be a viable method for solving power distribution system reconfiguration based on the radial nature of the distribution systems. The globally optimum configurations have been found for all considered test cases.

The improvement in power losses is significant and although the voltage profile was not directly controlled (cf. (1)), the optimized configurations exhibit significant improvement with this respect.

OpenDSS is used in this work to solve power flow, and although power flow method is different than that utilized by the benchmark methods, the numerical results are very close.

There are two features of FPEO that have to be emphasized. The first one is robustness. As opposed to majority of benchmark approaches, FPEO features excellent repeatability of results (globally optimum solutions obtained in all algorithm runs for 33- and 69-bus systems and all but one for 119-bus network). Second, the computational cost of the algorithm is dramatically lower than that of majority of the benchmark approaches. For example, the CSA, CGA, and PSO methods in [2] are set to 3000 function evaluations for 33- and 69-bus system, and 15000 evaluations for 119-bus system. FPEO works with 500, 1000 and 5000 maximum function evaluations for the 3 systems, respectively. In other words, FPEO offers a much faster convergence rate.

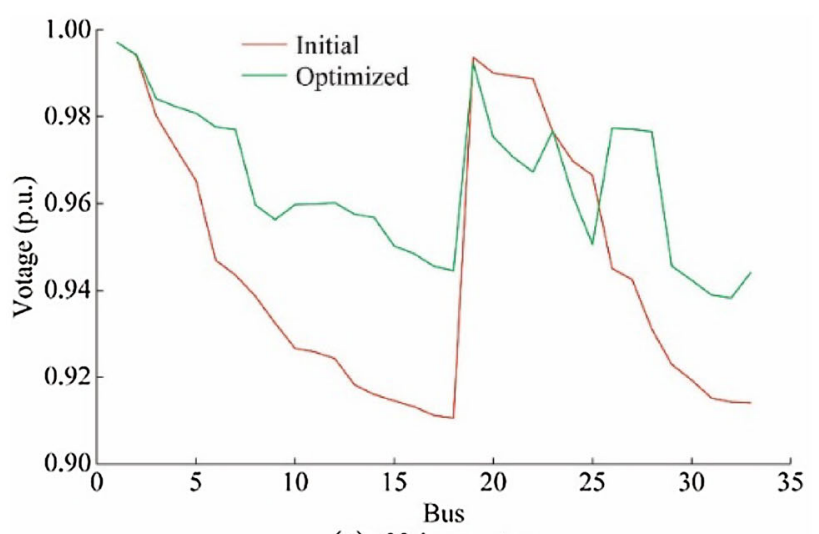

(a) 33-bus system

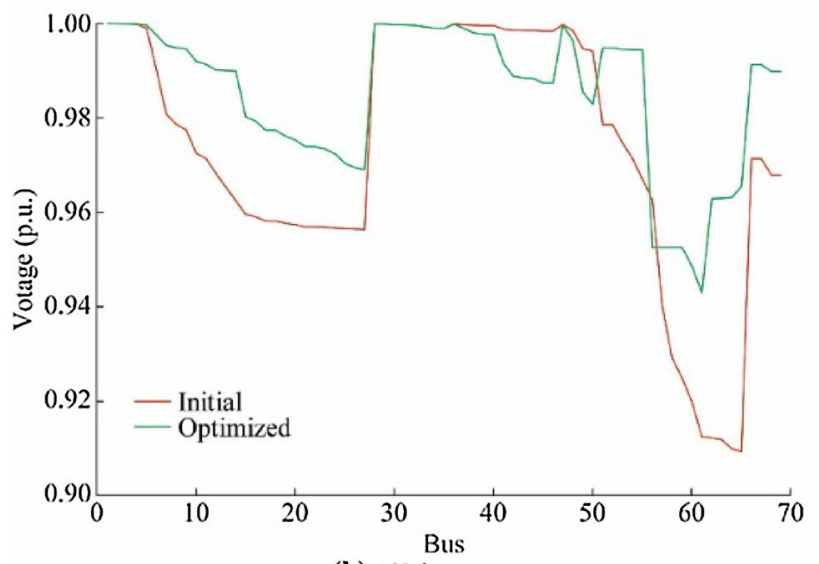

(b) 69-bus system

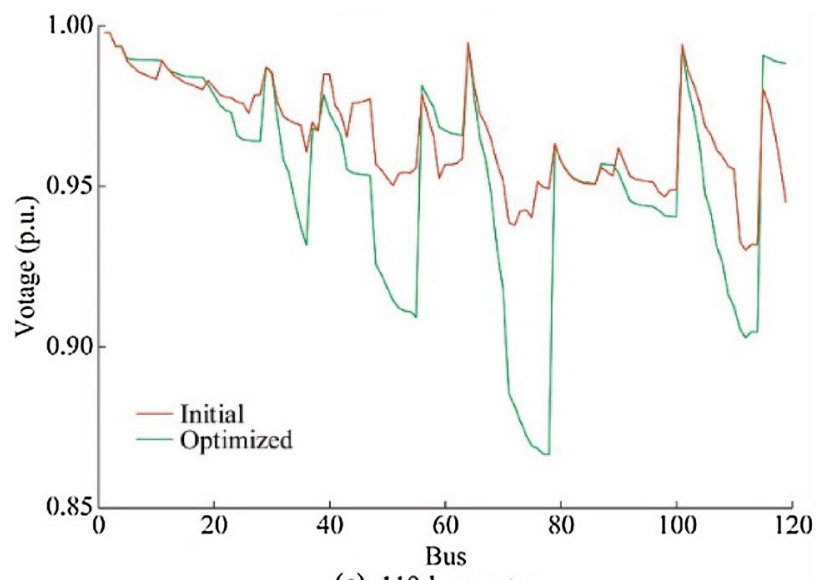

(c) 119-bus system

Fig. 12 Network voltage profile before and after reconfiguration/ optimization 


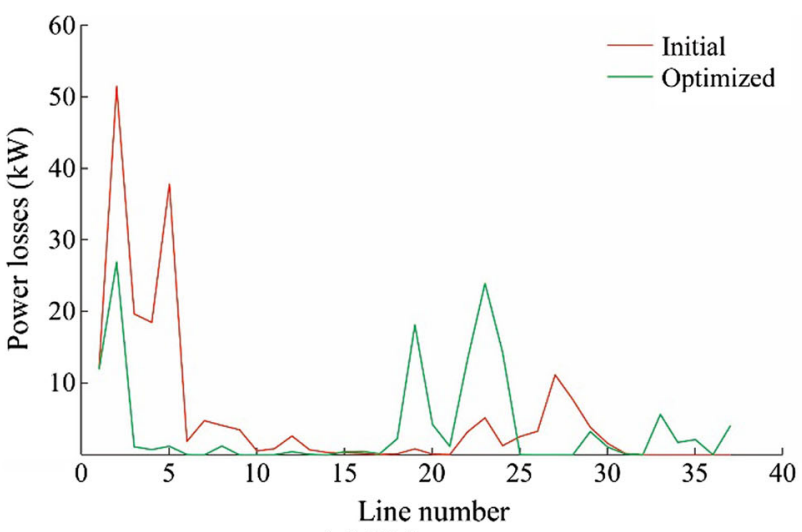

(a) 33-bus system

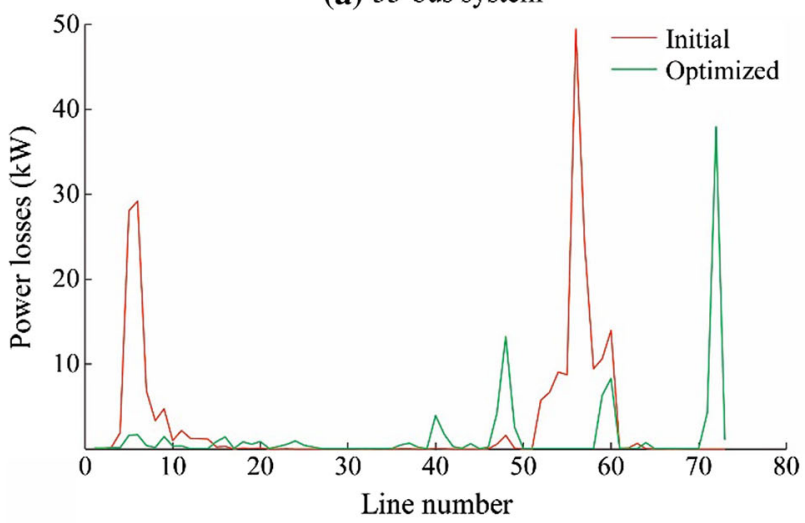

(b) 69-bus system

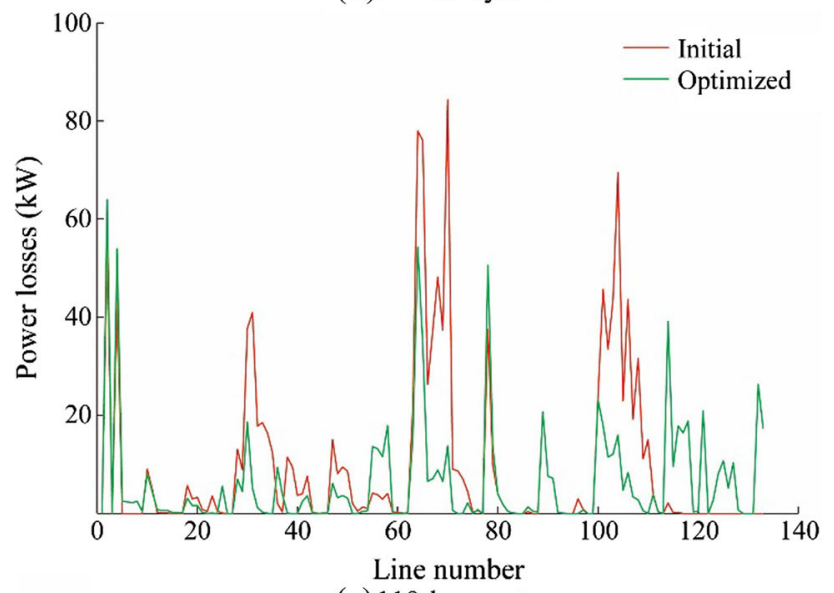

(c) 119-bus system

Fig. 13 Network power losses before and after reconfiguration/ optimization

\section{Conclusion}

In the paper, a customized evolutionary algorithm for solving distribution network reconfiguration problem has been presented. The proposed algorithm determines the optimized configuration of the network with respect to objectives being reduction of the power losses and improvement of the voltage profile.
The proposed algorithm features feasibility preserving mutation and recombination operators which allow us to maintain the radial structure of the network at all steps of the optimization process, which results in a dramatic reduction of the search space size.

As demonstrated, through comprehensive numerical validation, the proposed technique is superior over majority of the state-of-the-art methods reported in the literature. In particular, the computational cost of the optimization process is much lower than for most of competitive approaches. More importantly, repeatability of results is excellent, allowing for obtaining a globally optimum solution in almost each and every algorithm run.

The future work will be focused on extending the range of applications of the method, including multi-objective DNR (for both power loss reduction and voltage control), constrained optimization, as well as other types of networks (multi-source, non-radial).

Acknowledgements This work was supported in part by Mexico's National Council for Science and Technology-Sustentabilidad Energetica SENER CONACYT (2016) and National Science Centre of Poland Grant 2014/15/B/ST8/02315.

Open Access This article is distributed under the terms of the Creative Commons Attribution 4.0 International License (http:// creativecommons.org/licenses/by/4.0/), which permits unrestricted use, distribution, and reproduction in any medium, provided you give appropriate credit to the original author(s) and the source, provide a link to the Creative Commons license, and indicate if changes were made.

\section{References}

[1] Al-Mahroqi Y, Metwally IA, Al-Hinai A et al (2012) Reduction of power losses in distribution systems. World Acad Sci Eng Technol 63:585-592

[2] Nguyen TT, Truong AV (2015) Distribution network reconfiguration for power loss minimization and voltage profile improvement using cuckoo search algorithm. Int J Electr Power Energy Syst 68:233-242

[3] Ma Y, Liu F, Zhou X et al (2011) Overview on algorithms of distribution network reconfiguration. Power Syst Clean Energy 27(12):76-80

[4] Nie S, Fu XP, Li P (2012) Analysis of the impact of DG on distribution network reconfiguration using OpenDSS. In: Proceedings of IEEE PES innovative smart grid technologies, Tianjin, China, 21-24 May 2012, pp 1-5

[5] Merlin A, Back H (1975) Search for a minimal-loss operating spanning tree configuration in urban power distribution systems. In: Proceedings of $5^{\text {th }}$ power systems computational conference, Cambridge, UK, September 1975, pp 1-18

[6] Raju GKV, Bijwe PR (2008) Efficient reconfiguration of balanced and unbalanced distribution systems for loss minimisation. IET Gener Transm Distrib 2(1):7-12

[7] Gohokar VN, Khedkar MK, Dhole GM (2004) Formulation of distribution reconfiguration problem using network topology: a generalized approach. Electr Power Syst Res 69(2):305-310 
[8] Santos AC, Delbem ACB, London JBA et al (2011) Node-depth encoding and multiobjective evolutionary algorithm applied to large-scale distribution system reconfiguration. IEEE Trans Power Syst 25(3):1254-1265

[9] Imran AM, Kowsalya M (2014) A new power system reconfiguration scheme for power loss minimization and voltage profile enhancement using fireworks algorithm. Int J Electr Power Energy Syst 62:312-322

[10] Rao RS, Narasimham SVL, Raju MR et al (2011) Optimal network reconfiguration of large-scale distribution system using harmony search algorithm. IEEE Trans Power Syst 26(3):1080-1088

[11] Mirhoseini SH, Hosseini SM, Ghanbari M et al (2014) A new improved adaptive imperialist competitive algorithm to solve the reconfiguration problem of distribution systems for loss reduction and voltage profile improvement. Int J Electr Power Energy Syst 55:128-143

[12] Remolino AS, Paredes HFR (2016) An efficient method for power flow calculation applied to the reconfiguration of radial distribution systems. In: Proceedings of IEEE PES transmission and distribution conference and exposition-Latin America (PES T\&D-LA), Morelia, Mexico, 20-24 September 2016, pp 1-6

[13] Kersting WH (2001) Radial distribution test feeders. In: Proceedings of IEEE PES winter meeting, Columbus, USA, 28 January-1 February 2001, pp 908-912

[14] Yao YB, Wang D, Chen Y et al (2008) The effect of small impedance branches on the convergence of the Newton Raphson power flow. In: Proceedings of $3^{\text {rd }}$ international conference on electric utility deregulation and restructuring and power technologies, Nanjing, China, 6-9 April 2008, pp 1141-1146

[15] Dugan RC, McDermott TE (2011) An open source platform for collaborating on smart grid research. In: Proceedings of IEEE PES general meeting, San Diego, USA, 24-29 July 2011, pp 1-7

[16] Baran ME, Wu FF (1989) Network reconfiguration in distribution systems for loss reduction and load balancing. IEEE Trans Power Deliv 4(2):1401-1407

[17] Zhang D, Fu Z, Zhang L (2007) An improved TS algorithm for loss-minimum reconfiguration in large-scale distribution systems. Electr Power Syst Res 77:685-694

[18] Harris JM, Hirst JL, Mossinghoff MJ (2008) Combinatorics and graph theory, 2nd edn. Springer, New York

[19] Michalewicz Z (1996) Genetic algorithms + data structures $=$ evolutionary programs. Springer, New York

[20] Simon D (2013) Evolutionary optimization algorithms biologically inspired and population-based approaches to computer intelligence. Wiley, New York

[21] Chiang HD, Jean-Jumeau R (1990) Optimal network reconfigurations in distribution systems II: solution algorithms and numerical results. IEEE Trans Power Deliv 5(3):1568-1574
[22] Abdelaziz AY, Mohameda FM, Mekhamera SF et al (2010) Distribution system reconfiguration using a modified tabu search algorithm. Electr Power Syst Res 80(8):943-953

Alberto LANDEROS received the B.Sc. degree in energy engineering from Polytechnic University of Aguascalientes, Aguascalientes, Mexico, in 2015. He is currently working toward a M.Sc. degree in sustainable energy engineering-power systems at Reykjavik University, Iceland. He has done research in applied photovoltaics, solar power concentration, wind power, thermal power cycles, fluid dynamics, energy systems, high voltage conductors, underground conductors, corona effect and power systems. His current research interests include engineering optimization, numerical modeling, power systems, power system stability and control, transient simulations and machine learning. His employment experiences include Optics Research Center, Enersol Energy Solutions and Green Energy Geothermal.

Slawomir KOZIEL received the M.Sc. and Ph.D. degrees in electronic engineering from Gdansk University of Technology, Poland, in 1995 and 2000, respectively. He also received the M.Sc. degrees in theoretical physics and in mathematics, in 2000 and 2002, respectively, as well as the Ph.D. in mathematics in 2003, from the University of Gdansk, Poland. He is currently a professor with the School of Science and Engineering, Reykjavik University, Iceland. His research interests include CAD and modeling of microwave and antenna structures, simulation-driven design, surrogate-based optimization, space mapping, circuit theory, analog signal processing, evolutionary computation and numerical analysis.

Mohamed F. ABDEL-FATTAH received his Ph.D. degree in 2006 from Zagazig University, Egypt, after completing his B.Sc. and M.Sc. degrees, and then appointed as a Ph.D. lecturer with the Department of Electrical Power and Machines Engineering. He joined Aalto University, Finland, as a post-doctoral researcher and lecturer, with the power systems group at the Department of Electrical Engineering (2007-2009), and after that he joined the "smart grids and energy markets" project (2009-2014), for the development of the selfhealing capability of the smart distribution networks. Currently, he is an assistant professor at Reykjavik University, Iceland, with the School of Science and Engineering, since 2014. His research activities are mainly related to electrical power systems protection including earth-fault detection, diagnosis and location in medium and high-voltage networks, self-extinction property of arcing earth faults, development of the transient-based protection systems, and selfhealing for smart grids. 